\title{
Restoration of Population Disability Trajectory During Hundreds of Years - China, 1896-2006
}

\author{
Chao Guo ${ }^{1,2}$; Xiaoying Zheng ${ }^{1,2, \#}$
}

\begin{abstract}
Summary
What is already known about this topic?

A previous study found that the size of population with disability was 52.7 million and 84.6 million in 1987 and 2006 , with the weighted disability rate of $4.9 \%$ and $6.5 \%$, respectively.

What is added by this report?

This study restored the prevalence of disability and its change trajectory in China during 110 years from 1896 to 2006 across 3 centuries.

What are the implications for public health practice?

This study realized the innovation of cross-sectional data utilization methods and the expansion of the theory of morbidity transformation while making up for the lack of historical data.
\end{abstract}

With rapid changes of demographic structure and lifestyle, disability should be an important indicator of health evaluation and health promotion. However, due to the limitation of ideas and methods, research on the trend of disability is limited. Using data from the first and second China National Sample Survey on Disability (CNSSD) in 1987 and 2006, we restored the prevalence of population disability and its change trajectory in China during 110 years from 1896 to 2006 across 3 centuries through an innovative application of interdisciplinary methods. We found that since the end of the 19th century, the prevalence of disability among Chinese population presented a trend of fluctuating decreases, decreasing rapidly, and then increasing slowly. Three stages of evolution, which are closely related to the characteristics of social development and changes in mortality, were presented as dividing points in 1949 and 1986. The findings highlight that to further prevent and control disability and improve healthy life expectancy should be the main task and goal of health promotion.

Data used in this study were obtained from the first and second CNSSD in 1987 and 2006. Details about sampling, quality and key conceptions of the surveys can be found in our previous work (1-2). The two cross-sectional surveys contained the time of disability occurrence which was used as a key parameter to estimate the prevalence of disability in the previous 110 years. Data on total population, natural growth rate of population, mortality, etc., involved in calculations were derived from public information such as statistical yearbooks and literature.

Disability may occur under the combined effect of various risks of disability in the course of an individual's life from birth to death. At the population group level, the disability status of a population is a dynamic process consisting of new disabilities, disability rehabilitation, and death of the persons with disability. Thus, we established a flow chart of the disability process for a population as Figure 1 and considered simultaneous formulas with corresponding parameters to estimate the prevalence of disability in the previous 110 years since 2006 by mirroring reverse receding. The estimation process and details of calculation method were illustrated in Supplementary Figure S1 (available in weekly.chinacdc.cn).

Figure 2 shows the results of the restoration of population disability trajectory during 110 years from 1896-2006 across 3 centuries in China. In 1896, the starting point of this restoration, the prevalence of disability was nearly $55 \%$, and then gradually declined with relatively frequent fluctuations in a certain period. On the whole, since the end of the 19th century, the prevalence of population disability in China presented a trend of decreasing in fluctuation, decreasing rapidly and then increasing slowly.

Further observing the disability trajectory combined with changes of population mortality, we divided the development of population disability in China from 1896 to 2006 into 3 stages, with the dividing points of 1949 and 1986.

Stage I was from 1896 to 1948 , i.e., social upheavals and war years, during which the prevalence of disability of China declined from a high level in fluctuations, and the trend was opposite to that of mortality. Since it is difficult to obtain more accurate 


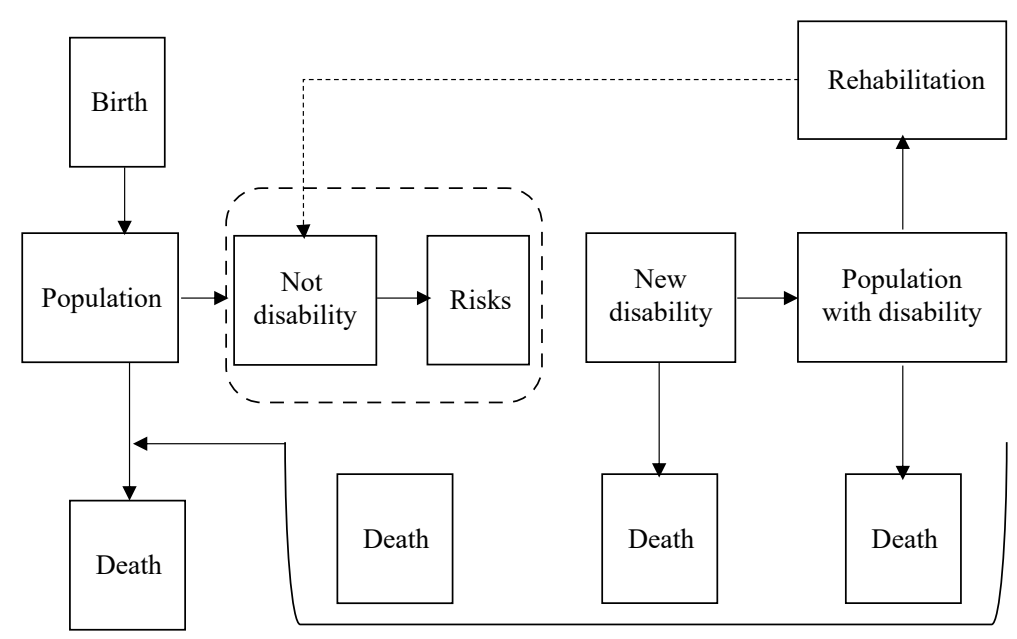

FIGURE 1. Flow chart of the disability process for a population.

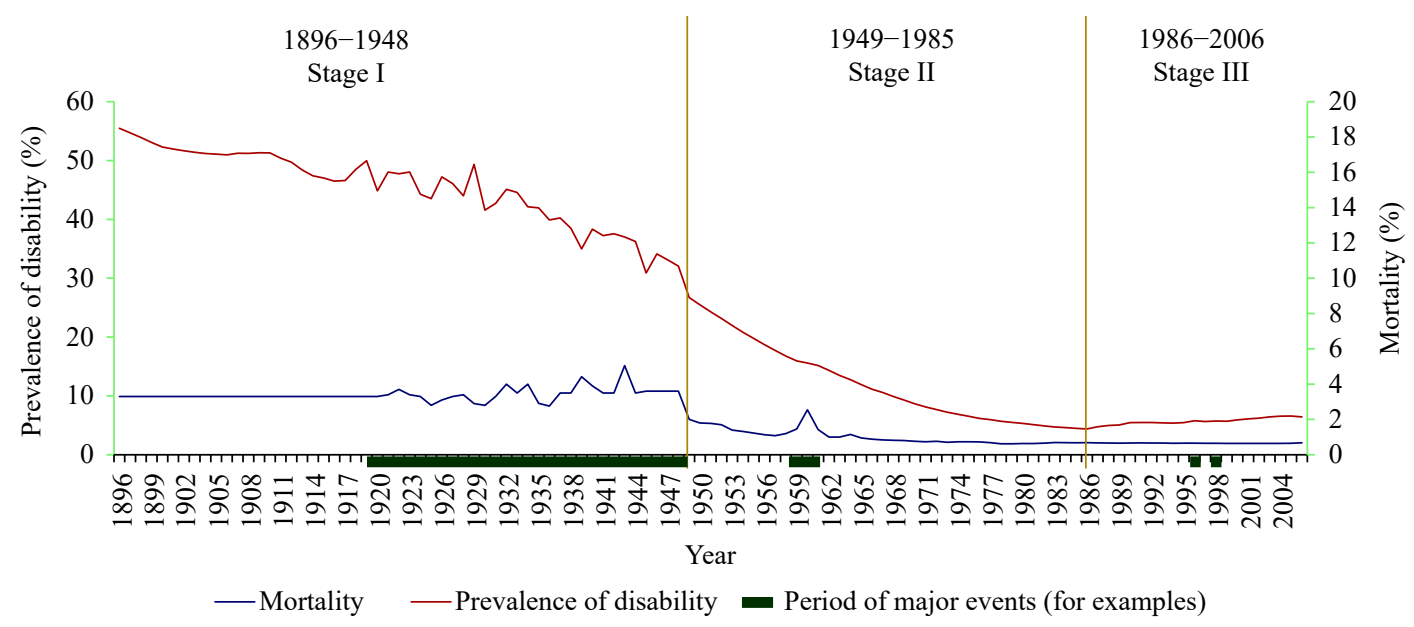

FIGURE 2. Restoration of population disability trajectory in China, 1896-2006.

Note: Major events for examples, a serise of wars during 1919-1948, the great famine during 1959-1961, earthquakes in 1996 and floods in 1998.

information on mortality in the early stages of history, we began to compare disability with mortality in Stage I since 1919, when relatively accurate figures of death were available. It is obvious from Figure 2 that during 1919-1948, both disability and mortality showed frequent fluctuations, and the "peaks and valleys" of the two generally fluctuated in the opposite direction.

Stage II was from 1949 to 1985 , i.e., the period of founding and early construction of new China, during which the prevalence of disability declined rapidly and showed the same trend as the mortality. And the decline of the prevalence of disability was faster than that of mortality.

Stage III was from 1986 to 2006, i.e., development and deepening of medical reform period, during which the prevalence of disability tended to rise and moved in the opposite direction of mortality. Both the rise of disability and decline of mortality were slow.

\section{DISCUSSION}

This study, for the first time, restores the evolutionary trajectory of the prevalence of disability during 110 years spanning 3 centuries from a historical perspective, which provides important information for the change of population health conditions and also contributes to the theory of morbidity transition and the methods of cross-sectional data use.

The 3 stages that we found in the disability trajectory during the 110 years confirmed the partial rationality of the relevant theory of "the compression of morbidity" (3) and "the expansion of morbidity" (4) with historical data and put forward supplementary ideas to further expand the theory. 
Specifically, Fries and Green pointed out that the compression of morbidity is the primary purpose of most health promotion activities in developed societies and there also remain opportunities to lessen mortality in developing societies (5). The characteristics of disability and mortality trends in Stage I and Stage II of our recovery trajectory indicated that the compression of morbidity could be also observed in relatively underdeveloped or developing societies. But its main driver is the low initial health endowment of the population in such societies. In this situation, individual health is vulnerable to risks. Thus, the impacts of risks on disability and death of population, especially on the mortality rate of population with disability, are also high. Therefore, although the prevalence of disability is high, the survival time of population with disability may be short, and the population reproduction or replacement in such societies should be rapid, which corresponds to the Stage I of our recovery trajectory. As we all know, China's social environment during this period was volatile and war-prone. The population were living with high mortality and low life expectancy at that time, with about 33 years in the Qing Dynasty and about 35 years in the Republican period (6). On the other hand, it could also cause the individual's life to change directly from a state of health to death without the appearance of an intermediate disability state when events such as wars break out, resulting in a "disabilitydeath substitution effect," which led to the opposite fluctuations of the two at this stage.

At the same time, however, the population in a developing society may be also sensitive to protective factors, and marginal health promotion may have a significant effect on the suppression of disability. However, due to the limited progress of medical technology, the rescue of death, especially the death of the persons with disability may not be improved at the same level, so the overall survival time of the population with disabilities is still short, forming a compensatory morbidity compression that most of the survival time is in a healthy state, which corresponds to Stage II of our recovery trajectory. This provides evidence to extend the theory of "the compression of morbidity" from the perspective of Chinese population health development.

The characteristics of Stage III in our recovery trajectory support the theory of "the expansion of morbidity." That is, when the technological progress of life saving exceeds the technological progress of health promoting, the proportion of living with disability in the total life span will increase (4). Moreover, the trends of disability and mortality changes is consistent with Wilson's hypothesis that the prevalence of disability will rise if the reduction of mortality is largely due to the rescue of death by medical technology ( 7 ). The social and medical development background at this stage in China also supports these theories. As we know, medical reform was conducted officially in China in 1985. With various reform measures, the pharmaceutical market, medical institutions, and research as well as medical workers have been activated rapidly, and the level of medical technology to save life have been rapidly improved.

Furthermore, the interaction between major natural and social events and population health inferred from theory also provides some support for the accuracy of our recovery trajectory. We highlighted some major events in Figure 2. As mentioned above, the frequent fluctuations of disability in Stage I coincide with the frequency of wars during 1919-1948. And we can observe an obvious "stagnation" in the rapid decline of the disability trajectory in Stage II during 1959-1961, when a great famine was affecting China, in line with the assumption of increasing disability. There were many earthquakes in China in 1996, especially earthquakes in Lijiang and Kunlun mountain with magnitude 7 or above; great floods attacked the Yangtze River, Nenjiang, and Songhua River in 1998. These natural disasters could partially explain the small peaks of disability in that two years shown in Stage III. Further studies on the relationship between major social and environmental events and population health under the framework of the events demography are also worthy of exploration through the details reflected by our recovery trajectory.

Unlike previous studies on forward predictions, this study obtains the results in historical periods by "reverse predictions". This is of more valuable academic importance since it is impossible to know a certain indicator in the past historical time if there is no record at that time. The reverse prediction of this study makes up for the lack of disability-related data in historical documents and also provides a scientific data platform for the precise prevention and control of disability. Especially, under the current situation of disability increase with mortality decrease, to further prevent and control disability and improve health life expectancy should be the main task and goal of health promotion. Additionally, by using the index "the time of disability occurrence" innovatively, this study deduced a long-term evolution trajectory from only 
two national cross-sectional surveys, which realizes the link and utilization of forgotten data, greatly improves the efficiency of data utilization and information acquisition, and provides a feasible technical method for the utilization of similar data.

This study was subject to several limitations. First, this study, to the best of our knowledge, is the first to explore the "reverse prediction," so the resources for reference in methods and comparison in results are both very limited and the findings need be further studied in the future. Second, we did not decompose the contribution of different types and causes of disability to the trend of disability changes. And the objective of our study is to demonstrate the development of disability, so we did not calculate the age-standardized prevalence to explore the demographic causes of this change. Moreover, since the first CNSSD was conducted in 1987, the development of screening and diagnosis could also contribute to the increase of disability in Stage III.

In conclusion, this study recovered the trajectory of disability change in population from 1896 to 2006 with only two national representative cross-sectional surveys. The findings of the transition law of Chinese population disability from the historical perspective emphasize a need for healthcare policy review to better serve population with disability in China, which may be applicable to other similar settings facing population health promotion against a background of population ageing and disability burden.
Acknowledgements: All the medical workers, investigators, and all relevant staff in the two CNSSDs.

Conflicts of interest: No conflicts of interest were reported.

Funding: National Social Science Foundation of China (No. 18CRK005).

doi: $10.46234 / \mathrm{ccdcw} 2021.121$

\# Corresponding author: Xiaoying Zheng, xzheng@pku.edu.cn.

${ }^{1}$ Institute of Population Research, Peking University, Beijing, China; ${ }^{2}$ APEC Health Science Academy (HeSAY), Peking University, Beijing, China.

Submitted: May 12, 2021; Accepted: May 22, 2021

\section{REFERENCES}

1. Zheng XY, Chen G, Song XM, Liu JF, Yan LJ, Du W, et al. Twentyyear trends in the prevalence of disability in China. Bull World Health Organ 2011;89(11):788 - 97. http://dx.doi.org/10.2471/BLT.11.089 730.

2. Guo C, Chang JH, Zheng XY, Wang LH. Utilization rate of healthcare service of the elderly with disabilities-China, 1987-2014. China CDC Wkly 2020;2(28):516 - 9. http://dx.doi.org/10.46234/ccdcw2020.140.

3. Fries JF. The compression of morbidity. Milbank Mem Fund Q Health Soc 1983;61(3):397 - 419. http://dx.doi.org/10.2307/3349864.

4. Gruenberg EM. The failures of success. Milbank Q 2005;83(4):779 800. http://dx.doi.org/10.1111/j.1468-0009.2005.00400.x.

5. Fries JF, Green LW, Levine S. Health promotion and the compression of morbidity. Lancet 1989;333(8636):481 - 3. http://dx.doi.org/10.1016/ S0140-6736(89)91376-7.

6. Lin WX. The average life span and life expectancy of all dynasties in China. Life Dis 1996(5): 27. http://www.cqvip.com/QK/97584B/ 199605/1004870865.html. (In Chinese).

7. Wilson RW. Do health indicators indicate health? Am J Public Health 1981;71(5):461 - 3. http://dx.doi.org/10.2105/AJPH.71.5.461. 


\section{Supplementary File}

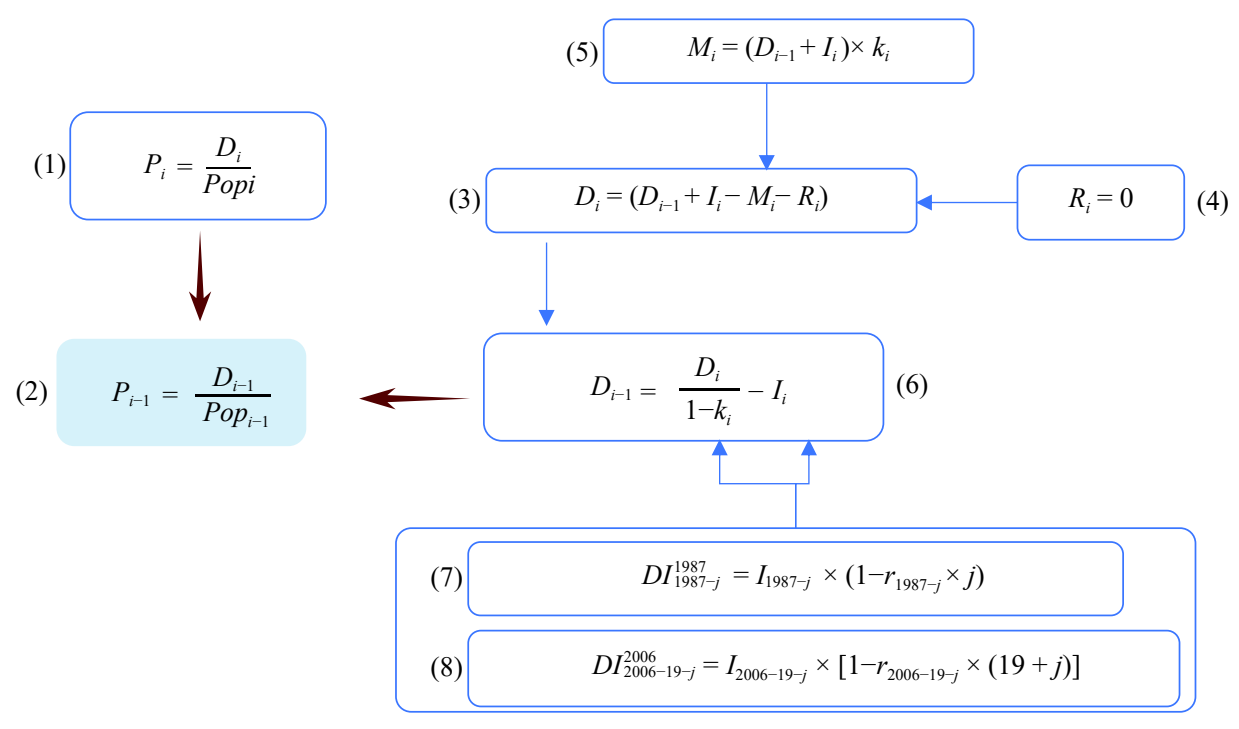

SUPPLEMENTARY FIGURE S1. The estimation process of the prevalence of disability.

Note: (1) $P_{i}$ denotes the prevalence of disability in year $i$, which is equal to the ratio of the size of persons with disability in year $i\left(D_{i}\right)$ to the total population in year $i\left(P o p_{i}\right)$. $P o p_{i}$ can be obtained from statistical yearbooks and literature.

(2). $P_{i-1}$ denotes the prevalence of disability in year $i-1$.

(3) $D_{i}$ is formed on the basis of persons with disability in the last year $i-1\left(D_{i-1}\right)$, by accumulating new persons with disabilities developed in year $i\left(I_{i}\right)$ and decaying deaths and rehabilitation of the persons with disabilities in that year.

(4) $R_{i}$ denotes persons with disabilities who recovered in year $i$. Since very few disabilities can be fully healed through rehabilitation and may only change the degree but not the identification of disability, we assume that $R_{i}$ is equal to 0 .

(5) $M_{i}$ denotes persons with disabilities who were dead in year $i$, including both those dead in $D_{i-1}$ and those in $I_{i}$; $k_{i}$ denotes the death rate of persons with disabilities.

(6) $D_{i-1}$ can be obtained by replacing the above parameters.

(7) \& (8). In China National Sample Survey on Disability China National Sample Survey on Disability 1987 and 2006, we can obtain $I_{1987}$ and $I_{2006}$. However, due to the presence of deaths of persons with disabilities, persons with disabilities who died before 1987 and 2006 were unable to report their time of disability occurrence during the survey window. Thus, the number of persons with disabilities reported in survey 1987 and 2006 occurred in years other than the year surveyed is not actually $I_{i}$, but is reduced by a certain rate of death $r$ from that year to 1987 or 2006.

$D I_{i}^{1987}$ and $D I_{i}^{2006}$ denote persons with disabilities reported in survey 1987 and 2006 occurred in year $i$, and persons with disabilities occurred in year 1913-2006 were both reported in survey 1987 and 2006. Then, $r$ and $I$ during 1913-1987 can be calculated from equations (7) and (8). We assumed that $k$ during 1913-1987 was linearly altered from $r_{1913}$ to $r_{1987}$, and $k$ and $r$ during 1890-1912 and during 1988-2005 were equal to $r_{1913}$ and $r_{1987}$, respectively. Then $D_{i}$ from 2006 to the year before can then be obtained. 